\title{
Pathogenesis of chronic obstructive pulmonary disease (COPD) induced by cigarette smoke
}

\author{
Mari Hikichi, Kenji Mizumura, Shuichiro Maruoka, Yasuhiro Gon \\ Division of Respiratory Medicine, Department of Internal Medicine, Nihon University School of Medicine, Tokyo, Japan \\ Contributions: (I) Conception and design: All authors; (II) Administrative support: All authors; (III) Provision of study materials or patients: All \\ authors; (IV) Collection and assembly of data: All authors; (V) Data analysis and interpretation: All authors; (VI) Manuscript writing: All authors; (VII) \\ Final approval of manuscript: All authors. \\ Correspondence to: Mari Hikichi, MD, PhD. Division of Respiratory Medicine, Department of Internal Medicine, Nihon University School of \\ Medicine, 30-1 Oyaguchi Kamicho, Tokyo, Japan. Email hikichi.mari@nihon-u.ac.jp.
}

\begin{abstract}
Chronic obstructive pulmonary disease (COPD) is a common respiratory disease that is characterized by functional and structural alterations primarily caused by long-term inhalation of harmful particles. Cigarette smoke (CS) induces airway inflammation in COPD, which is known to persist even after smoking cessation. This review discusses the basic pathogenesis of COPD, with particular focus on an endogenous protective mechanism against oxidative stress via $\mathrm{Nrf} 2$, altered immune response of the airway inflammatory cells, exaggerated cellular senescence of the lung structural cells, and cell death with expanded inflammation. Recently, CS-induced mitochondria autophagy is reported to initiate programmed necrosis (necroptosis). Necroptosis is a new concept of cell death which is driven by a defined molecular pathway along with exaggerated inflammation. This new cell death mechanism is of importance due to its ability to produce more inflammatory substances during the process of epithelial death, contributing to persistent airway inflammation that cannot be explained by apoptosis-derived cell death. Autophagy is an auto-cell component degradation system executed by lysosomes that controls protein and organelle degradation for successful homeostasis. As well as in the process of necroptosis, autophagy is also observed during cellular senescence. Aging of the lungs results in the acquisition of senescence-associated secretory phenotypes (SASP) that are known to secrete inflammatory cytokines, chemokines, growth factors, and matrix metalloproteinases resulting in chronic low-grade inflammation. In future research, we intend to highlight the genetic and epigenetic approaches that can facilitate the understanding of disease susceptibility. The goal of precision medicine is to establish more accurate diagnosis and treatment methods based on the patientspecific pathogenic characteristics. This review provides insights into CS-induced COPD pathogenesis, which contributes to a very complex disease. Investigating the mechanism of developing COPD, along with the availability of the particular inhibitors, will lead to new therapeutic approaches in COPD treatment.
\end{abstract}

Keywords: Airway inflammation; autophagy; cellular senescence; chronic obstructive pulmonary disease (COPD); necroptosis; oxidative stress

Submitted Sep 22, 2019. Accepted for publication Oct 20, 2019.

doi: $10.21037 /$ jtd.2019.10.43

View this article at: http://dx.doi.org/10.21037/jtd.2019.10.43

\section{Introduction}

The lungs are a complex organ comprising numerous cells that are continually exposed to infectious agents, cigarette smoke (CS), and pollutants. The disruption of homeostasis in response to constant levels of harmful inhalants leads to irreversible morphological and functional alterations in the lungs.

The molecular pathology underlying chronic obstructive pulmonary disease (COPD) is influenced by genetic 


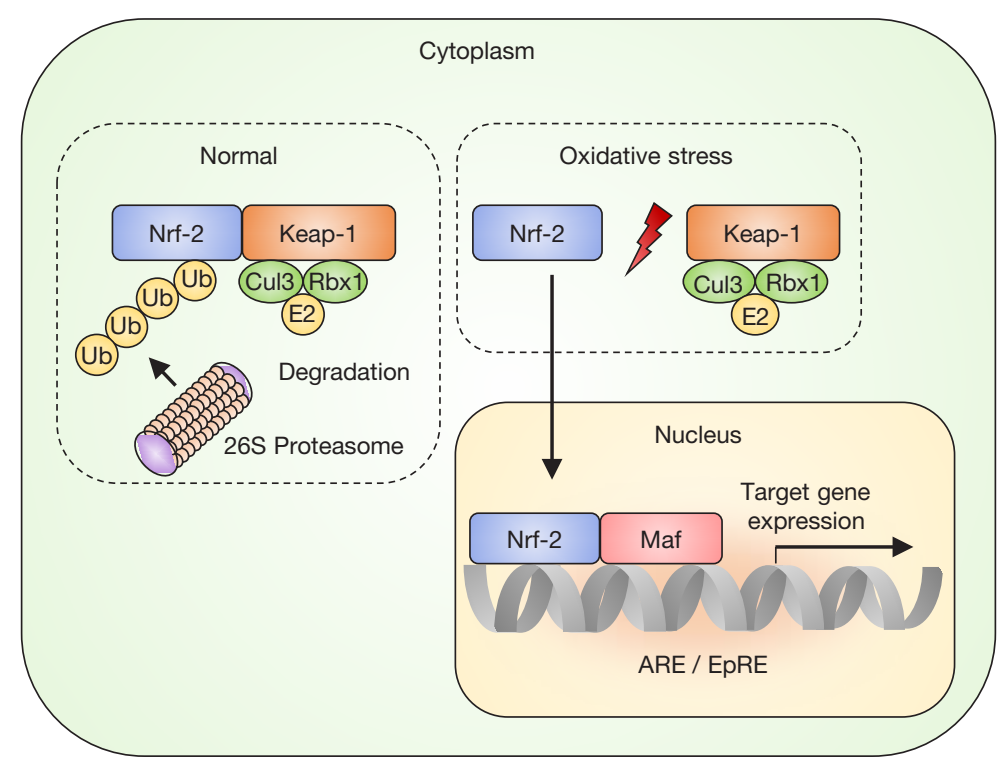

Figure 1 Keap-1 and Nrf-2 pathways. Under normal conditions, Nrf-2 is localized in the cytoplasm and is bound to Keap-1. Keap-1 can also combine with cul3 and Rbx1 to form the core ubiquitin 3 ligase complex, which results in ubiquitination and proteasomal degradation of Nrf-2. Under oxidative stress conditions, Nrf-2 and Keap-1 dissociate, which allows Nrf-2 to translocate to the nucleus, where it forms a heterodimer with Maf and binds to ARE to induce the gene expression of antioxidant and detoxifying enzymes. Nrf-2, nuclear factorerythroid 2 related factor 2; Keap-1, Kelch-like ECH-associated protein 1; Cul3, culin-3; Rbx1, ring box 1; ARE, antioxidant response element; EpRE, electrophile responsive element.

background, cellular senescence, and chronic inhalation of harmful particles, such as those present in CS. Toxic particles of inhaled smoke induce airway inflammation that is exacerbated in COPD patients; such chronic inflammation is known to persist even after smoking cessation (1). CS contains numerous toxic chemical substances (2), including oxygen-derived metabolites or reactive oxygen species (ROS) which play an essential role in antimicrobial activity, cell signaling, and other mechanisms necessary for maintaining homeostasis. However, excessive accumulation of ROS leads to harmful modifications in proteins, lipids, and DNA (3). Oxidative stress markers, such as lipid peroxides, including hydrogen peroxide, nitric oxide, and 8 -isoprostane, as well as nitrotyrosine and nitrogen oxides, are elevated in the respiratory tract, lungs, and blood of COPD patients (3).

In this review, we provide an overview of airway inflammation, morphological changes, and cellular senescence observed in the lungs during CS-induced COPD, with a focus on aberrant autophagy and cell death, which are new and important concepts in COPD pathogenesis.

\section{Protection from oxidative stress in the lungs}

In healthy lungs, antioxidants, such as superoxide dismutase, catalase, and glutathione peroxidase, provide an endogenous biological defense against oxidative stress caused by CS. An imbalance between the oxidants and antioxidants leads to increased expression of genes involved in inflammation, increased secretion of airway mucus, and inactivation of anti-proteases (3).

Nuclear factor E2-related factor 2 (Nrf2) facilitates antioxidant gene expression (4). Under non-stressed conditions, Nrf2 is retained in the cytoplasm by direct binding to the Kelch-like ECH-associated protein 1 (Keap1) (Figure 1). The Keap1 gene also binds to cullin-3-ring box 1 to form the core ubiquitin E3 ligase complex. Consequently, Keap1-bound Nrf2 is ubiquitinated and degraded by the $26 \mathrm{~S}$ proteasome that prevents its accumulation in the cytoplasm $(4,5)$. In contrast, ROS impairs the bonding of Keap1-Nrf2 and allows Nrf2 to translocate into the nucleus where it escapes the homeostatic protein degradation. In the nucleus, Nrf2 heterodimerizes with small Maf proteins and binds to the antioxidant-responsive or electrophileresponsive elements. Thus, the transcription of target genes, 


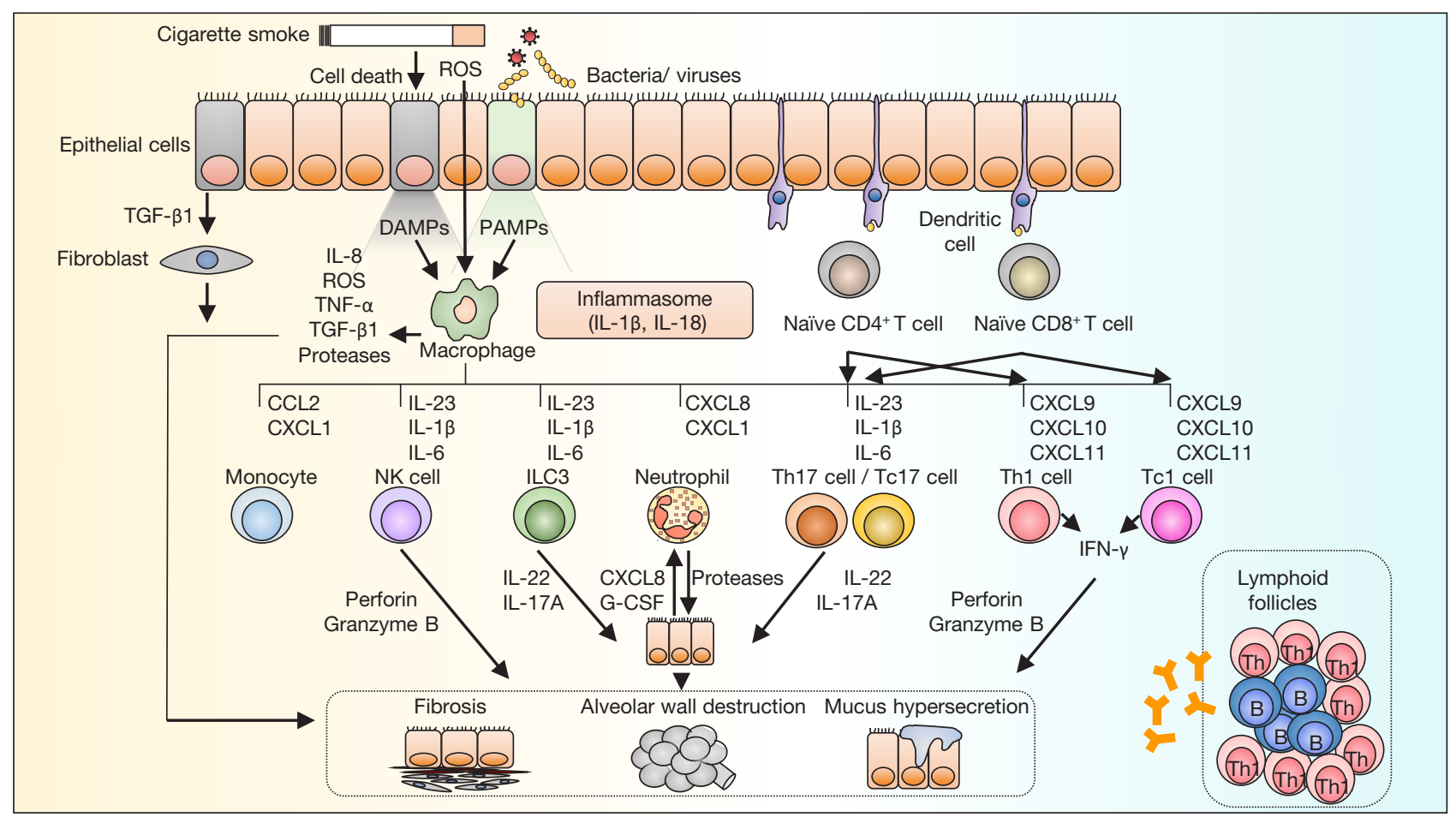

Figure 2 Airway inflammation in COPD. Inhaled irritants activate epithelial cells and alveolar macrophages, which play a central role in type 1 airway inflammation by releasing cytokines and chemokines. Alveolar macrophages and neutrophils release proteases, such as MMPs and neutrophil elastase, which cause elastin degradation that results in alveolar wall destruction. Dendritic cells are an important link between innate immunity and adaptive immunity, locating near the epithelium surface to sense the entry of inhaled irritants. ROS, reactive oxygen species; DAMPs, damage-associated molecular patterns; PAMPs, pathogen-associated molecular patterns; TNF- $\alpha$, tumor necrosis factor- $\alpha$; IL, interleukin; TGF- $\beta 1$, transforming growth factor- $\beta 1$; CCL, C-C motif chemokine; CXCL, chemokine (C-X-C motif) ligand; NK cell, natural killer cell; ILC3, innate lymphoid cell 3; Th, helper T lymphocyte; Tc, cytotoxic T lymphocyte; G-CSF, granulocyte colony-stimulating factor; IFN- $\gamma$, interferon-gamma; MMPs, matrix metalloproteinases; COPD, chronic obstructive pulmonary disease.

such as those encoding antioxidant proteins and xenobioticmetabolizing enzymes, is activated to mount a biological defense $(4,5)$.

More than 500 genes are regulated by the Nrf2/ antioxidant-responsive element signaling, including target genes of oxidative stress (heme oxygenase, $\gamma$-glutamyl cysteine ligase), inflammation [transforming growth factor $\beta$ (TGF- $\beta$ ) and nuclear factor (NF)-кB], xenobiotic metabolism (nicotinamide adenine dinucleotide phosphate quinone oxidoreductase, aldo-keto reductase family 1 member $\mathrm{C} 1$, and multidrug resistance-associated protein 1), apoptosis (BCL2 and BCL-XL), and autophagy (p62) (6). Therefore, the genes involved in antioxidant and antiinflammatory responses, xenobiotic metabolism, detoxification, and anti-proliferation are expressed downstream of the Nrf2 pathway $(6,7)$.
Significant evidence shows that oxidative stress damages the lungs and contributes to COPD pathogenesis. It is possible that therapeutic administration of multiple antioxidants will be effective in the management of COPD (8-10).

\section{Immunity in COPD}

\section{Activation of innate immunity}

Inhaled particles and pathogens are recognized with pattern recognition receptors (PRRs) on the plasma membrane of the alveolar epithelial cells and macrophages (Figure 2). There are several types of PRRs: toll-like receptors, cytosolic NOD-like receptor (NLRs), and RIG-I-like receptors (11). PRRs are activated by damage-associated molecular patterns (DAMPs) released after tissue damage, 
resulting in the production of inflammatory cytokines via a caspase 1-dependent mechanism and the formation of the nucleotide-binding oligomerization domain like receptor (NLR) P3 inflammasome. NLRP3 inflammasome leads to the secretion of interleukin (IL)-1 $\alpha$, IL-1 $\beta$, IL-33, and IL-18 $(12,13)$. The induced IL-1 $\beta$ and IL-18 activate neutrophils, macrophages, helper T (Th) 1, and Th17 lymphocytes leading to Type1 airway inflammation $(12,13)$. Research has shown that inflammasome-dependent cytokines are involved in COPD pathogenesis; however evidence that NLRP3 inflammasome is indeed driving COPD pathogenesis remains inconclusive. Increased expression of NLRP3 and IL-1 $\beta$ are observed in COPD lungs $(14,15)$. CSexposed NLRP3 knock-out mice exhibit decrease production of caspase-1, IL-1 $\beta$ and IL-18 (16). Apoptosis-associated speck-like protein containing a caspase recruitment domain (ASC) are found to play an important role in inflammasome to recruit pro-caspase-1. ASC specks get phagocytosed by macrophages and promote the production of IL- $1 \beta$ by these cells (17). In patients with COPD, ASC specks are found abundant in bronchoalveolar lavage (BAL) fluid and peripheral blood mononuclear cells (17). In contrast, Di Stefano et al. examined the expression of NLRP3 inflammasome-associated factors in bronchial mucosa and BAL fluid collected from patients with stable COPD (18). Interestingly, they found that no increment in the levels of NLRP3, caspase-1 and IL-1 $\beta$ observed in patients with COPD when compared to control smokers (18). Pauwels et al. reported that CS-induced pulmonary inflammation was independent of NLRP3 inflammasome or caspase-1 deficiency (19), indicating the inflammation was mediated in a NLRP3-independent manner. Some randomized clinical trials that target inflammasome-relating effectors have been performed on COPD patients, but did not present positive results $(20,21)$. Several studies support the involvement of NLRP3 inflammasome in the pathogenesis of COPD, there are still controversies in this field that need further investigation.

Alveolar macrophages play a central role in airway inflammation. These cells secrete multiple chemokines and cytokines, such as tumor necrosis factor- $\alpha(\mathrm{TNF}-\alpha)$, that induce the expression of adhesion molecules on endothelial cells, facilitating the migration of a variety of inflammatory cells (Figure 2) (22). Alveolar macrophages also produce ROS, metalloproteinases (MMPs), and cathepsins to disrupt alveolar structures and induce fibrosis mediators such as TGF- $\beta 1$ to trigger airway remodeling (22).

In COPD, neutrophil apoptosis is inhibited and phagocytic function of alveolar macrophages that treat apoptotic neutrophils are decreased. This leads to neutrophil-induced inflammation by causing secondary necrosis (23). The phagocytic function of alveolar macrophages can be improved by sulforaphane via Nrf2 signaling (24).

Several recent studies have focused on the various macrophage phenotypes in COPD (25). Macrophages metabolize $\mathrm{L}$-arginine to nitric oxide via inducible nitric oxide synthase (iNOS) or to ornithine via arginase-1. M1 macrophages express iNOS and secrete pro-inflammatory cytokines, such as interferon $\gamma$ and IL-22, to mediate type 1 inflammation (26,27). In contrast, M2 macrophages express arginase- 1 and secrete Th2 cytokines, such as IL-4, IL-13, and IL-10, to mediate tissue fibrosis, perform repair, and create an anti-inflammatory response in the airways (28). Eapen et al. reported that healthy nonsmokers harbored macrophages that were not polarized to the M1 or M2 phenotype. In contrast, patients with COPD and healthy smokers harbored higher numbers of M1 macrophages in the small airway walls and M2 macrophages in the BAL fluid. This shows the difference in the macrophage polarity between the interior and exterior of the airway walls (29). It is noteworthy that macrophages in the BAL fluid samples collected from COPD patients exhibited increased expression in Th2 cytokines (IL-4, IL-13, and IL-5) and decreased expression in interferon $\gamma$, suggesting impaired protection from infections (29).

Neutrophils are an important component of type 1 inflammation. Elevated neutrophils levels in sputum is associated with COPD severity (30). Neutrophil migration to the lungs is caused by the accumulation of ROS inducedphosphatidylinositol 3,4,5-triphosphate at the injury site (31). In addition, chemokine C-X-C motif ligand (CXCL) 1, CXCL8, and leukotriene $\mathrm{B} 4$ produced by macrophages and IL-22, IL-17A produced by Th, lymphocytes and innate lymphoid cell (ILC) $3(32,33)$ are involved in the determination of neutrophil motility. Airway neutrophils secrete myeloperoxidase, neutrophil elastase (NE), cathepsins, proteinase, and MMPs and are directly involved in the destruction of alveoli and promote mucus production in the submucosal glands and goblet cells (34-38). These proteolytic substance and neutrophilic DNA with dimensions as small as $2 \mathrm{~nm}$ consist neutrophil extracellular traps (NETs). NETs play an important role in host defense against infection by trapping microbes that are too large to be involved in the phagocytosis process and are potentially cytotoxic (39). A recent report reveals that CS induces NET 
component. In stable COPD patients, abundant NETs are observed in the sputum, correlating with severity of airflow limitation (40). In the innate immunity process, LL-37 and $\alpha$ defensins, the intracellular components of NETs, promote NLRP3 inflammasome in response to CS (41).

\section{The shift toward adaptive immunity}

Antigens released by cell and tissue injury are recognized by the dendritic cells and presented to T-lymphocytes to activate adaptive immunity. CS-induced NETs can enhance plasmacytoid dendritic cells, generating naive CD4-positive $\mathrm{T}$ cells into Th1 and Th17 (42). In healthy subjects, regulatory T (Treg) cells, M2 macrophages, and myeloidderived suppressor cells create immune tolerance that suppresses immune activation to facilitate a shift toward adaptive immunity (43). This process is impaired in COPD, enabling the development of adaptive immune response (43).

Treg cells are a subset of CD4-positive T lymphocytes that play a role in immune suppression. Naturally occurring Treg cells differentiate functionally in the thymus, while inducible Treg cells differentiate from naïve $\mathrm{T}$ lymphocytes in the periphery. TGF- $\beta$ is required for the differentiation of Treg cells, with FoxP3 acting as the master transcription factor (44). Treg cells are believed to suppress excessive immune response by producing the inhibitory cytokines TGF- $\beta 1$ and IL-10 and expressing the cytotoxic T lymphocyte-associated protein 4 (44). In COPD, the Th17/ Treg ratios in the blood and sputum are shifted toward the Th17 cells; further, the absence of an anti-inflammatory response in the respiratory tract is considered to contribute to persistent airway inflammation in COPD $(45,46)$. Furthermore, CD8-positive cytotoxic T lymphocytes and CD4-positive Th1 lymphocytes as well as natural killer cells comprise the innate immunity and release perforin and granzyme B to induce apoptosis of the epithelial cells, followed by alveolar destruction $(22,47)$. Although the molecular mechanisms of amplified airway inflammation in COPD remain unclear, alteration in the innate and adaptive immune response could be a possible explanation.

\section{Morphological change of the airway epithelium}

Chronic exposure to CS directly affects the morphology and function of the airway epithelium. Impaired epithelial function and shortening of the cilia on the epithelium are observed through histone deacetylase 6-dependent, autophagy-mediated mechanisms induced by CS $(48,49)$.
The reduction in mucociliary clearance leads to goblet cell hyperplasia that affects the epithelial microenvironment $(34,50)$. In COPD lungs, decreased activity of Nrf2 is reported (51,52). The importance of Nrf2 is confirmed by CS-exposed Nrf2-deficient mice demonstrating a development of emphysematous lesion in the lungs (53).

Adherens and tight junctions comprise the epithelial barrier that limits the invasion of the inhaled harmful particles. CS-mediated ROS has been shown to disrupt the tight junctions via an epidermal growth factor receptordependent mechanism (54). Moreover, ROS breaks hyaluronic acid into small particles, $70 \mathrm{kDa}$ in size, allowing these particles to bind to the layilin receptor and activate the Rho-associated protein kinase. This ultimately leads to the reduction in E-cadherin gene expression and the disruption of the adherens junctions (55). The disruption of the epithelial barrier and the reduction in E-cadherin expression induces epithelial-mesenchymal transition, consequently inducing the aberrant production of MMPs and growth factors, airway destruction, and remodeling $(56,57)$. These alterations in structural cells are important for understanding the mechanism of morphological changes of the lungs and various symptoms present in COPD.

\section{Cellular senescence and cell-repair mechanisms}

The incidence of COPD is high among the elderly. During healthy aging, reduced elasticity and alveolar dilation are accompanied by low-level inflammation; however, these changes are not associated with emphysematous changes or the destruction of the alveolar walls observed in the lung tissue samples of COPD patients (58). These age-related alterations are abnormally increased in COPD patients owing to several reasons, including telomere shortening, cellular senescence, activation of the phosphatidylinositol-3-kinase $(\mathrm{PI} 3 \mathrm{~K}) / \mathrm{mammalian}$ target of rapamycin (mTOR) signaling pathway, defective DNA repair, abnormal microRNA patterns, epigenetic alterations, decreased anti-aging molecules, mitochondrial dysfunction, impaired autophagy, immunosenescence, and stem cell exhaustion $(59,60)$.

The most probable explanation for the age-associated changes in COPD is the intracellular and extracellular production of ROS. Appropriate ROS levels are necessary for normal aging, while excessive ROS levels lead to cell cycle arrest and cell senescence via activation of the tumor suppressor protein p53 (60). The PI3K/AKT/mTOR pathway is at the core of the accelerated aging observed in COPD (Figure 3). PI3K is activated by ROS, followed by 


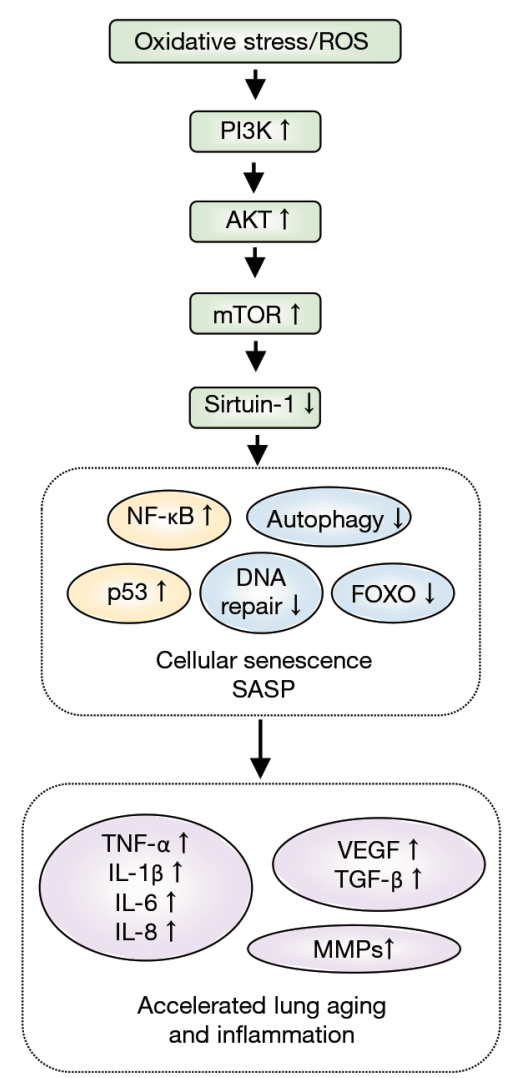

Figure 3 Mechanisms of accelerated aging and inflammation in COPD. Oxidative stress reduces sirtuin-1 through PI3K/ AKT/mTOR signaling. Decreased sirtuin-1 results in cellular senescence. Acquisition of SASP, result in accelerated lung aging and inflammation. ROS, reactive oxygen species; PI3K, phosphatidylinositol-3 kinase; mTOR, mammalian target of rapamycin; NF- $\kappa \mathrm{B}$, nuclear factor- $\kappa \mathrm{B}$; FOXO, Forkhead box $\mathrm{O}$; TNF- $\alpha$, tumor necrosis factor- $\alpha$; IL, interleukin; TGF, transforming growth factor; VEGF, vascular endothelial growth factor; MMPs, matrix metalloproteinases; SASP, senescenceassociated secretory phenotype; COPD, chronic obstructive pulmonary disease.

the activation of AKT and mTOR, with the subsequent inhibition of sirtuin-1 (SIRT1). SIRT1 is a histone deacetylase that regulates numerous transcription factors and is closely related to oxidative stress, inflammation, and cellular senescence (61). Compared to apoptotic cells, senescent cells produce numerous inflammatory proteins which can affect other tissues and cells. These senescent cells are referred as senescence-associated secretory phenotype (SASP) (62). SASP is activated by p21, leading to the activation of $\mathrm{p} 38$ mitogen-activated protein kinase and Janus-activated kinase. This is followed by the release of inflammatory cytokines (IL- $1 \beta$, IL- 6 , and TNF- $\alpha$ ), growth factors (vascular endothelial growth factor, TGF- $\beta 1$ ), chemokines, and MMPs, downstream from $\mathrm{NF}-\kappa \mathrm{B}$ activation (63). Furthermore, SASP contributes to the activation of the NRLP3 inflammasome that induces prolonged, low-grade airway inflammation (64). Studies have also reported decreased expression of SIRT1 and increased expression of cellular senescence markers in the lung tissue samples of COPD patients and the airway epithelial cells exposed to CS $(61,65,66)$.

Healthy lungs possess regenerative capacity and can replace injured airway epithelial cells with new epithelial cells differentiated from the airway basal progenitor cells. However, the basal progenitor cells in the lungs of COPD patients are reduced in number and have lost the selfrenewal ability and multipotentiality. Moreover, basal and mucus goblet cells are most common amongst postdifferentiated cells with decreased ciliated cells, which contributes to COPD pathogenesis after tissue repair (67). Dysfunction of basal progenitor cells correlates with COPD severity (67).

Cellular senescence also occurs in stem cells. In normal stem cells, the preservation of a quiescent state is essential to maintain replication competence. Many stem cells prevent oxidative phosphorylation by mitochondria and are often dependent on metabolism via the anaerobic glycolytic pathway to avoid cellular senescence induced by the intracellularly produced ROS. In the presence of excessive ROS, activated proliferation in the stem cells leads to further production of ROS that results in cellular senescence and loss of replication competence (68). Senescence of the mesenchymal stem cells (fibroblasts and endothelial cells) and alveolar type II cells that are differentiated into alveolar type I cells are reported to develop emphysematous lesions and COPD $(69,70)$. Thus, accelerated aging in the lungs of COPD patients results in the secretion of inflammatory proteins and loss of lung repairing system.

\section{A new cell death concept in COPD}

\section{Necroptosis: a new concept of regulated necrosis}

Emphysematous changes in COPD have mainly been discussed in the context of apoptosis of lung epithelial cells, wherein cell death occurs with a limited release of DAMPs in the absence of an inflammatory response. The molecular pathogenesis of COPD is characterized by airway 
inflammation; however, evidence on the relationship between airway inflammation and epithelial apoptosis is lacking. For decades, necrosis was considered as an accidental form of cell death that did not require any specific molecular signaling pathways. Recent studies have demonstrated that a certain degree of necrosis is a well-designed form of cell death; the receptor-interacting protein kinase (RIPK) 3-dependent and caspase-independent programmed necrosis is currently defined as necroptosis (71). Compared to apoptosis, necroptosis is considered to be a type of programmed cell death involving greater DAMPs release and inflammatory response (72).

However, several upstream signaling elements of apoptosis and necroptosis are shared, and sensitivity to each death pathway is regulated by the overlapping cluster of regulatory molecules (71). One of the most well-analyzed necroptosis pathway starts from TNF receptor (TNFR) 1 activation. After TNF- $\alpha$ binding, TNFR1 binds to various molecules including caspase 8 and RIPK1. RIPK1 ubiquitination leads to the activation of mitogen-activated protein kinase and $\mathrm{NF}-\mathrm{\kappa B}$ signaling, resulting in inflammatory response and cell survival. While activation of caspase 8 leads to apoptosis, inactivation of caspase 8 results in initiating necroptosis. In necroptosis, RIPK1 forms an intracellular complex with RIPK3 to assemble the necrosome-an amyloidlike structure that serves as a necroptotic signal transducer. RIPK3 phosphorylates mixed lineage kinase domain-like protein (MLKL) and translocates phosphorylated MLKL to the cell membrane, resulting in direct pore formation with DAMP release $(73,74)$.

Pouwels et al. revealed that CS-induced necroptosis and the release of DAMPs trigger neutrophilic inflammation in mice that was reduced with a necroptosis inhibitor, necrostatin-1 (75). Moreover, Wang et al. reported that endoplasmic reticulum chaperone GRP78 promoted a CSinduced inflammatory response and mucus hyperproduction in airway epithelial cells. This may occur through the upregulation of necroptosis and subsequent activation of the NF- $\mathrm{KB}$ and activator protein-1 pathways (76), indicating that necroptosis playing a crucial role in COPD development.

\section{Autophagy}

Recent studies have focused on the involvement of autophagy in COPD. Autophagy is an umbrella term that defines an auto-cell component degradation system executed by the lysosomes. In various models, the notion of autophagy as induced by cell starvation is shown to be a pro-survival process during nutrient shortage, with the cells undergoing partial self-degradation and recycling (77). Recently, autophagy has been detected not only in response to starvation stimuli, but also in conditions of stress and hormonal changes that reflect its significance in the progression and regulation of multiple disease processes (78).

Autophagy is regulated by a complex signaling network of various nutrient signals (amino acids and glucose), growth factors (insulin), and inhibitory inputs. mTOR plays a central role in autophagy by its inhibition. Autophagy is upregulated in the circumstance of energy charge depletion, and is associated with a suppression of mTOR complex 1. Execution of autophagy is initiated with nucleation and phagophore formation regulated by beclin-1. This is followed by the elongation of the nascent autophagosomal membrane to capture cargos, such as organelles and proteins. Finally, following the maturation of the doublemembrane autophagosomal structure, the autophagosome fuses with the lysosome and degrades autolysosomal contents (79-81).

Previous studies that have analyzed comprehensive gene expression profiles in Global Initiative for Chronic Obstructive Lung Disease stage 2 versus stage 0 smokers have shown that microtubule-associated protein 1 light chain (LC) 3, the autophagosome molecule, may be a potential molecular target in COPD (82). Lung tissue samples of COPD patients and alveolar epithelial cells exposed to CS extracts exhibit increased expression of LC3B-II, leading to the Fas-mediated induction of apoptosis through the activation of autophagy (83). In addition, CSinduced emphysema was suppressed in mice knocked out for $L c 3 b$ or beclin1, two important regulators of autophagy (83); thus, the activation of autophagy was correlated with COPD pathogenesis. However, these findings do not explain the specific details on how autophagy regulates CS-induced cell death.

Autophagy was previously considered to act as a nonselective self-degradation system; however, accumulating evidence indicates the presence of selective autophagy that includes the selective degradation of specific organelles and pathogens. Recently, autophagy that selectively targets the mitochondria (mitophagy) is reported to regulate cellular senescence in COPD. The protein levels of mitophagy markers are decreased in COPD lungs compared with non-COPD lungs (84). Genetically inhibiting mitophagy resulted in an enhancement of CS-induced mitochondrial ROS production and cellular senescence in airway epithelial cells (84). 


\section{The relationship between necroptosis and autophagy in COPD}

Autophagy has recently been redefined in the framework of "cell death accompanied by autophagy" instead of "cell death induced by autophagy" (85); this has left the issue regarding the contribution of autophagy to cell death unresolved.

Mizumura et al. demonstrated the role of mitophagy in COPD pathogenesis (72). In this study, exposure to CS extract caused significant mitochondrial depolarization and induced mitophagy in pulmonary epithelial cells $(72,85)$. A mitophagy inhibitor, Mdivi-1, reduced phosphorylation of MLKL in the necroptosis pathway and showed protection against CS-induced cell death. PTEN induced kinase 1 (PINK1) is a mitophagy regulator. CS-exposed PINK1deficient mice showed a reduction in mitochondrial dysfunction as well as protection against airspace enlargement and mucociliary clearance disruption. This result suggested that activation of mitophagy may alter mitochondrial membrane integrity and lead to necroptosis in experimental COPD model (72).

There are many other factors that impact cell death in COPD. A recent report indicates sphingolipid metabolism in CS exposure-related mitophagy and necroptosis may be involved (86). CS-exposed murine models exhibit accumulation of iron causing programmed necrotic cell death (ferroptosis), resulting in an airspace enlargement and small airway thickness in the lungs $(87,88)$.

Cell death patterns are becoming a growing interest in the pathogenesis of COPD. Understanding the mechanism of cell death signaling, along with the availability of inhibitors, is now focused as a new therapeutic target in COPD.

\section{Future directions: genetic approaches for understanding disease susceptibility}

In this review, we describe CS-induced molecular pathogenesis in COPD. However, there is a difference in the individual responsiveness to CS exposure. While CS remains the most important risk factor among COPD patients, life-long smokers only have 10-20\% lifetime incidence of COPD, indicating the importance of genetic susceptibility (89). Advances in genome-wide association study (GWAS) have resulted in the discovery of genetic variants and traits via the detection of singlenucleotide polymorphisms. Major COPD GWAS include
ECLIPSE, COPDGene and ICGN cohorts (90). The COPDGene is one of the largest studies to investigate the underlying genetic factors and natural history of COPD. With the enrollment of over 10,000 smokers with or without COPD since 2008, COPDGene aims to characterize disease-related phenotypes and explore the associations with susceptibility genes by assessing symptom scores, comorbidities, pulmonary function, imaging, biomarker, and genetic profiling data $(91,92)$. This ongoing longitudinal evaluation asks subjects to return for the third follow-up visit after enrollment. Recent large-scaled studies have revealed multiple causal genes and related potential therapeutic biological pathways $(93,94)$. Recently, there are advances in gene editing technologies to analyze the function of the target gene. Upcoming pioneer tools for genome editing include zinc finger nucleases, transcription activator-like effector nucleases and clustered regulatory interspaced short palindromic repeats/CRISPR-associated protein 9 technologies as well as conventional homologous recombination (95). Furthermore, numerous studies have provided evidence of epigenetic modification (DNA modification, histone modification, and non-coding RNAs) in the pathogenesis of COPD (95). DNA methylation of C10orf11, a known COPD-associated gene identified via GWAS, was observed in the airway epithelial cells and lung tissues of smokers who develop COPD (96). Several recent reports have suggested microRNAs as potential biomarkers because they affect the expression of the target genes associated with structural and inflammatory alternations in the lungs (97-101). Although further studies are needed to explore the clinical application, research focusing on disease susceptibility needs to be conducted to prevent COPD development and identify potential therapeutic targets.

\section{Conclusions}

In this review, we have provided an overview of the molecular pathophysiological insights of oxidative stress, airway inflammation, cellular senescence, and cell death caused by CS in COPD. Many studies have found that CS-induced excessive ROS damage the lungs and contribute to COPD pathogenesis. Nrf2 protects the lungs against oxidative stress, while decrease activity of Nrf2 results in insufficient production of antioxidant molecules. Oxidative process not only affects the initiation process of lung injury, but also induces cellular senescence in the epithelium cells and stem cells. Lung ageing causes acquisition of SASP that secrete inflammatory cytokines, chemokines, growth factors, and 
MMPs. Altered immune response in the airways causes prolonged inflammation and structural modifications. The relationship between autophagy and programmed necrosis (necroptosis) involved in CS-induced cell death is a novel finding that reveals the mechanism of exaggerated inflammatory response that is not explained by apoptosisderived cell death. Finally, we described the genetic approaches for understanding disease susceptibility in the development of COPD.

COPD is a complex disease with various molecular pathogenic mechanisms. A comprehensive understanding of the heterogeneity in COPD and its etiology is required in the clinical setting. Successful preventive, diagnostic, and therapeutic outcomes will closely depend on accurate evaluation of the underlying pathogenesis. Further research is needed to develop strategies for therapeutic intervention in COPD.

\section{Acknowledgments}

None.

\section{Footnote}

Conflicts of Interest: The authors have no conflicts of interest to declare.

Ethical Statement: The authors are accountable for all aspects of the work in ensuring that questions related to the accuracy or integrity of any part of the work are appropriately investigated and resolved.

\section{References}

1. Wang Y, Xu J, Meng Y, et al. Role of inflammatory cells in airway remodeling in COPD. Int J Chron Obstruct Pulmon Dis 2018;13:3341-8.

2. Pryor WA, Stone K. Oxidants in cigarette smoke. Radicals, hydrogen peroxide, peroxynitrate, and peroxynitrite. Ann N Y Acad Sci 1993;686:12-27; discussion 27-8.

3. McGuinness AJ, Sapey E. Oxidative Stress in COPD: Sources, Markers, and Potential Mechanisms. J Clin Med 2017. doi: 10.3390/jcm6020021.

4. Abed DA, Goldstein M, Albanyan H, et al. Discovery of direct inhibitors of Keap1-Nrf2 protein-protein interaction as potential therapeutic and preventive agents. Acta Pharm Sin B 2015;5:285-99.

5. Zhao H, Eguchi S, Alam A, et al. The role of nuclear factor-erythroid 2 related factor 2 (Nrf-2) in the protection against lung injury. Am J Physiol Lung Cell Mol Physiol 2017;312:L155-62.

6. Liu Q, Gao Y, Ci X. Role of Nrf2 and Its Activators in Respiratory Diseases. Oxid Med Cell Longev 2019;2019:7090534.

7. Rojo AI, Sagarra MR, Cuadrado A. GSK-3 $\beta$ downregulates the transcription factor $\mathrm{Nrf} 2$ after oxidant damage: relevance to exposure of neuronal cells to oxidative stress. J Neurochem 2008;105:192-202.

8. Tse HN, Raiteri L, Wong KY, et al. Benefits of highdose $\mathrm{N}$-acetylcysteine to exacerbation-prone patients with COPD. Chest 2014;146:611-23.

9. Vlahos R, Bozinovski S. Glutathione peroxidase-1 as a novel therapeutic target for COPD. Redox Rep 2013;18:142-9.

10. Rahman I. Antioxidant therapeutic advances in COPD. Ther Adv Respir Dis 2008;2:351-74.

11. Kawai T, Akira S. The roles of TLRs, RLRs and NLRs in pathogen recognition. Int Immunol 2009;21:317-37.

12. Brightling C, Greening N. Airway inflammation in COPD: progress to precision medicine. Eur Respir J 2019;54.

13. Scambler T, Holbrook J, Savic S, et al. Autoinflammatory disease in the lung. Immunology 2018. [Epub ahead of print].

14. Faner R, Sobradillo P, Noguera A, et al. The inflammasome pathway in stable COPD and acute exacerbations. ERJ Open Res 2016;2(3).

15. Kim RY, Pinkerton JW, Gibson PG, et al. Inflammasomes in COPD and neutrophilic asthma. Thorax 2015;70:1199-201.

16. Eltom S, Belvisi MG, Stevenson CS, et al. Role of the inflammasome-caspase1/11-IL-1/18 axis in cigarette smoke driven airway inflammation: an insight into the pathogenesis of COPD. PLoS One 2014;9:e112829.

17. Franklin BS, Bossaller L, De Nardo D, et al. The adaptor ASC has extracellular and 'prionoid' activities that propagate inflammation. Nat Immunol 2014;15:727-37.

18. Di Stefano A, Caramori G, Barczyk A, et al. Innate immunity but not NLRP3 inflammasome activation correlates with severity of stable COPD. Thorax 2014;69:516-24.

19. Pauwels NS, Bracke KR, Dupont LL, et al. Role of IL1alpha and the Nlrp3/caspase-1/IL-1beta axis in cigarette smoke-induced pulmonary inflammation and COPD. Eur Respir J 2011;38:1019-28.

20. Calverley PMA, Sethi S, Dawson M, et al. A randomised, 
placebo-controlled trial of anti-interleukin-1 receptor 1 monoclonal antibody MEDI8968 in chronic obstructive pulmonary disease. Respir Res 2017;18:153.

21. Rogliani P, Calzetta L, Ora J, et al. Canakinumab for the treatment of chronic obstructive pulmonary disease. Pulm Pharmacol Ther 2015;31:15-27.

22. Barnes PJ. Inflammatory mechanisms in patients with chronic obstructive pulmonary disease. J Allergy Clin Immunol 2016;138:16-27.

23. Hoenderdos K, Condliffe A. The neutrophil in chronic obstructive pulmonary disease. Am J Respir Cell Mol Biol 2013;48:531-9.

24. Harvey CJ, Thimmulappa RK, Sethi S, et al. Targeting Nrf2 signaling improves bacterial clearance by alveolar macrophages in patients with COPD and in a mouse model. Sci Transl Med 2011;3:78ra32.

25. Wojtan P, Mierzejewski M, Osinska I, et al. Macrophage polarization in interstitial lung diseases. Cent Eur J Immunol 2016;41:159-64.

26. Mills CD. Anatomy of a discovery: $\mathrm{m} 1$ and $\mathrm{m} 2$ macrophages. Front Immunol 2015;6:212.

27. Mills CD, Kincaid K, Alt JM, et al. M-1/M-2 macrophages and the Th1/Th2 paradigm. J Immunol 2000;164:6166-73.

28. Martinez FO, Gordon S. The M1 and M2 paradigm of macrophage activation: time for reassessment. F1000Prime Rep 2014;6:13.

29. Eapen MS, Hansbro PM, McAlinden K, et al. Abnormal M1/M2 macrophage phenotype profiles in the small airway wall and lumen in smokers and chronic obstructive pulmonary disease (COPD). Sci Rep 2017;7:13392.

30. Noguera A, Batle S, Miralles C, et al. Enhanced neutrophil response in chronic obstructive pulmonary disease. Thorax 2001;56:432-7.

31. Kuiper JW, Sun C, Magalhaes MA, et al. Rac regulates PtdInsP(3) signaling and the chemotactic compass through a redox-mediated feedback loop. Blood 2011;118:6164-71.

32. Ardain A, Porterfield JZ, Kloverpris HN, et al. Type 3 ILCs in Lung Disease. Front Immunol 2019;10:92.

33. De Grove KC, Provoost S, Verhamme FM, et al. Characterization and Quantification of Innate Lymphoid Cell Subsets in Human Lung. PLoS One 2016;11:e0145961.

34. Shaykhiev R. Emerging biology of persistent mucous cell hyperplasia in COPD. Thorax 2019;74:4-6.

35. Hao W, Li M, Zhang Y, et al. Expressions of MMP12, TIMP-4, and Neutrophil Elastase in PBMCs and Exhaled Breath Condensate in Patients with COPD and Their Relationships with Disease Severity and Acute
Exacerbations. J Immunol Res 2019;2019:7142438.

36. Hou HH, Wang HC, Cheng SL, et al. MMP-12 activates protease-activated receptor-1, upregulates placenta growth factor, and leads to pulmonary emphysema. Am J Physiol Lung Cell Mol Physiol 2018;315:L432-42.

37. Gupta V, Khan A, Higham A, et al. The effect of phosphatidylinositol-3 kinase inhibition on matrix metalloproteinase-9 and reactive oxygen species release from chronic obstructive pulmonary disease neutrophils. Int Immunopharmacol 2016;35:155-62.

38. Churg A, Zhou S, Wright JL. Series "matrix metalloproteinases in lung health and disease": Matrix metalloproteinases in COPD. Eur Respir J 2012;39:197-209.

39. Twaddell SH, Baines KJ, Grainge C, et al. The Emerging Role of Neutrophil Extracellular Traps in Respiratory Disease. Chest 2019;156:774-82.

40. Grabcanovic-Musija F, Obermayer A, Stoiber W, et al. Neutrophil extracellular trap (NET) formation characterises stable and exacerbated COPD and correlates with airflow limitation. Respir Res 2015;16:59.

41. Wright TK, Gibson PG, Simpson JL, et al. Neutrophil extracellular traps are associated with inflammation in chronic airway disease. Respirology 2016;21:467-75.

42. Qiu SL, Zhang H, Tang QY, et al. Neutrophil extracellular traps induced by cigarette smoke activate plasmacytoid dendritic cells. Thorax 2017;72:1084-93.

43. Cosio MG, Saetta M. Evasion of COPD in smokers: at what price? Eur Respir J 2012;39:1298-303.

44. Tang Q, Bluestone JA. The Foxp3+ regulatory T cell: a jack of all trades, master of regulation. Nat Immunol 2008;9:239-44.

45. Li H, Liu Q, Jiang Y, et al. Disruption of th17/treg balance in the sputum of patients with chronic obstructive pulmonary disease. Am J Med Sci 2015;349:392-7.

46. Li XN, Pan X, Qiu D. Imbalances of Th17 and Treg cells and their respective cytokines in COPD patients by disease stage. Int J Clin Exp Med 2014;7:5324-9.

47. Hashimoto S, Kobayashi A, Kooguchi K, et al. Upregulation of two death pathways of perforin/granzyme and FasL/Fas in septic acute respiratory distress syndrome. Am J Respir Crit Care Med 2000;161:237-43.

48. Cloonan SM, Lam HC, Ryter SW, et al. "Ciliophagy": The consumption of cilia components by autophagy. Autophagy 2014;10:532-4.

49. Lam HC, Cloonan SM, Bhashyam AR, et al. Histone deacetylase 6-mediated selective autophagy regulates COPD-associated cilia dysfunction. J Clin Invest 2013;123:5212-30. 
50. Schamberger AC, Mise N, Jia J, et al. Cigarette smokeinduced disruption of bronchial epithelial tight junctions is prevented by transforming growth factor-beta. Am J Respir Cell Mol Biol 2014;50:1040-52.

51. Ishii Y, Itoh K, Morishima Y, et al. Transcription factor Nrf2 plays a pivotal role in protection against elastaseinduced pulmonary inflammation and emphysema. J Immunol 2005;175:6968-75.

52. Suzuki M, Betsuyaku T, Ito Y, et al. Down-regulated NFE2-related factor 2 in pulmonary macrophages of aged smokers and patients with chronic obstructive pulmonary disease. Am J Respir Cell Mol Biol 2008;39:673-82.

53. Rangasamy T, Cho CY, Thimmulappa RK, et al. Genetic ablation of Nrf2 enhances susceptibility to cigarette smoke-induced emphysema in mice. J Clin Invest 2004;114:1248-59.

54. Petecchia L, Sabatini F, Varesio L, et al. Bronchial airway epithelial cell damage following exposure to cigarette smoke includes disassembly of tight junction components mediated by the extracellular signal-regulated kinase $1 / 2$ pathway. Chest 2009;135:1502-12.

55. Forteza RM, Casalino-Matsuda SM, Falcon NS, et al. Hyaluronan and layilin mediate loss of airway epithelial barrier function induced by cigarette smoke by decreasing E-cadherin. J Biol Chem 2012;287:42288-98.

56. Milara J, Peiro T, Serrano A, et al. Epithelial to mesenchymal transition is increased in patients with COPD and induced by cigarette smoke. Thorax 2013;68:410-20.

57. Sohal SS, Walters EH. Epithelial mesenchymal transition (EMT) in small airways of COPD patients. Thorax 2013;68:783-4.

58. Fukuchi Y. The aging lung and chronic obstructive pulmonary disease: similarity and difference. Proc Am Thorac Soc 2009;6:570-2.

59. Barnes PJ. Senescence in COPD and Its Comorbidities. Annu Rev Physiol 2017;79:517-39.

60. Mercado N, Ito K, Barnes PJ. Accelerated ageing of the lung in COPD: new concepts. Thorax 2015;70:482-9.

61. Conti V, Corbi G, Manzo V, et al. Sirtuin 1 and aging theory for chronic obstructive pulmonary disease. Anal Cell Pathol (Amst) 2015;2015:897327.

62. Parikh P, Wicher S, Khandalavala K, et al. Cellular senescence in the lung across the age spectrum. Am J Physiol Lung Cell Mol Physiol 2019;316:L826-42.

63. Barnes PJ, Baker J, Donnelly LE. Cellular Senescence as a Mechanism and Target in Chronic Lung Diseases. Am J Respir Crit Care Med 2019;200:556-64.
64. Acosta JC, Banito A, Wuestefeld T, et al. A complex secretory program orchestrated by the inflammasome controls paracrine senescence. Nat Cell Biol 2013;15:978-90.

65. Yao H, Chung S, Hwang JW, et al. SIRT1 protects against emphysema via FOXO3-mediated reduction of premature senescence in mice. J Clin Invest 2012;122:2032-45.

66. Birch J, Anderson RK, Correia-Melo C, et al. DNA damage response at telomeres contributes to lung aging and chronic obstructive pulmonary disease. Am J Physiol Lung Cell Mol Physiol 2015;309:L1124-37.

67. Ghosh M, Miller YE, Nakachi I, et al. Exhaustion of Airway Basal Progenitor Cells in Early and Established Chronic Obstructive Pulmonary Disease. Am J Respir Crit Care Med 2018;197:885-96.

68. Ito K, Suda T. Metabolic requirements for the maintenance of self-renewing stem cells. Nat Rev Mol Cell Biol 2014;15:243-56.

69. Chilosi M, Carloni A, Rossi A, et al. Premature lung aging and cellular senescence in the pathogenesis of idiopathic pulmonary fibrosis and COPD/emphysema. Transl Res 2013;162:156-73.

70. Barkauskas CE, Cronce MJ, Rackley CR, et al. Type 2 alveolar cells are stem cells in adult lung. J Clin Invest 2013;123:3025-36.

71. Linkermann A, Green DR. Necroptosis. N Engl J Med 2014;370:455-65.

72. Mizumura K, Cloonan SM, Nakahira K, et al. Mitophagydependent necroptosis contributes to the pathogenesis of COPD. J Clin Invest 2014;124:3987-4003.

73. Wang L, Du F, Wang X. TNF-alpha induces two distinct caspase-8 activation pathways. Cell 2008;133:693-703.

74. Dondelinger Y, Aguileta MA, Goossens V, et al. RIPK3 contributes to TNFR1-mediated RIPK1 kinase-dependent apoptosis in conditions of cIAP1/2 depletion or TAK1 kinase inhibition. Cell Death Differ 2013;20:1381-92.

75. Pouwels SD, Zijlstra GJ, van der Toorn M, et al. Cigarette smoke-induced necroptosis and DAMP release trigger neutrophilic airway inflammation in mice. Am J Physiol Lung Cell Mol Physiol 2016;310:L377-86.

76. Wang Y, Zhou JS, Xu XC, et al. Endoplasmic reticulum chaperone GRP78 mediates cigarette smoke-induced necroptosis and injury in bronchial epithelium. Int J Chron Obstruct Pulmon Dis 2018;13:571-81.

77. Mizushima N, Komatsu M. Autophagy: renovation of cells and tissues. Cell 2011;147:728-41.

78. Choi AM, Ryter SW, Levine B. Autophagy in human health and disease. N Engl J Med 2013;368:651-62. 
79. Mizumura K, Cloonan S, Choi ME, et al. Autophagy: Friend or Foe in Lung Disease? Ann Am Thorac Soc 2016;13 Suppl 1:S40-7.

80. Racanelli AC, Kikkers SA, Choi AMK, et al. Autophagy and inflammation in chronic respiratory disease. Autophagy 2018;14:221-32.

81. Yang Z, Klionsky DJ. Eaten alive: a history of macroautophagy. Nat Cell Biol 2010;12:814-22.

82. Ning W, Li CJ, Kaminski N, et al. Comprehensive gene expression profiles reveal pathways related to the pathogenesis of chronic obstructive pulmonary disease. Proc Natl Acad Sci U S A 2004;101:14895-900.

83. Chen $\mathrm{ZH}$, Lam HC, Jin Y, et al. Autophagy protein microtubule-associated protein 1 light chain-3B (LC3B) activates extrinsic apoptosis during cigarette smoke-induced emphysema. Proc Natl Acad Sci U S A 2010;107:18880-5.

84. Ito S, Araya J, Kurita Y, et al. PARK2-mediated mitophagy is involved in regulation of HBEC senescence in COPD pathogenesis. Autophagy 2015;11:547-59.

85. Ballweg K, Mutze K, Konigshoff M, et al. Cigarette smoke extract affects mitochondrial function in alveolar epithelial cells. Am J Physiol Lung Cell Mol Physiol 2014;307:L895-907.

86. Mizumura K, Justice MJ, Schweitzer KS, et al. Sphingolipid regulation of lung epithelial cell mitophagy and necroptosis during cigarette smoke exposure. FASEB J 2018;32:1880-90.

87. Yoshida M, Minagawa S, Araya J, et al. Involvement of cigarette smoke-induced epithelial cell ferroptosis in COPD pathogenesis. Nat Commun 2019;10:3145.

88. Cloonan SM, Glass K, Laucho-Contreras ME, et al. Mitochondrial iron chelation ameliorates cigarette smokeinduced bronchitis and emphysema in mice. Nat Med 2016;22:163-74.

89. Pérez-Rubio G, Cordoba-Lanus E, Cupertino P, et al. Role of genetic susceptibility in nicotine addiction and chronic obstructive pulmonary disease. Rev Invest Clin 2019;71:36-54.

90. Kan M, Shumyatcher M, Himes BE. Using omics approaches to understand pulmonary diseases. Respir Res

Cite this article as: Hikichi M, Mizumura K, Maruoka S, Gon Y. Pathogenesis of chronic obstructive pulmonary disease (COPD) induced by cigarette smoke. J Thorac Dis 2019;11(Suppl 17):S2129-S2140. doi: 10.21037/jtd.2019.10.43
2017;18:149.

91. Regan EA, Hokanson JE, Murphy JR, et al. Genetic epidemiology of COPD (COPDGene) study design. Copd 2010;7:32-43.

92. Maselli DJ, Bhatt SP, Anzueto A, et al. Clinical Epidemiology of COPD: Insights From 10 Years of the COPDGene Study. Chest 2019;156:228-38.

93. Shrine N, Guyatt AL, Erzurumluoglu AM, et al. New genetic signals for lung function highlight pathways and chronic obstructive pulmonary disease associations across multiple ancestries. Nat Genet 2019;51:481-93.

94. Sakornsakolpat P, Prokopenko D, Lamontagne M, et al. Genetic landscape of chronic obstructive pulmonary disease identifies heterogeneous cell-type and phenotype associations. Nat Genet 2019;51:494-505.

95. Wadhwa R, Aggarwal T, Malyla V, et al. Identification of biomarkers and genetic approaches toward chronic obstructive pulmonary disease. J Cell Physiol 2019;234:16703-23.

96. Morrow JD, Cho MH, Hersh CP, et al. DNA methylation profiling in human lung tissue identifies genes associated with COPD. Epigenetics 2016;11:730-9.

97. Donaldson A, Natanek SA, Lewis A, et al. Increased skeletal muscle-specific microRNA in the blood of patients with COPD. Thorax 2013;68:1140-9.

98. Burke H, Heinson A, Freeman A, et al. Late Breaking Abstract - Differentially expressed exosomal miRNAs target key inflammatory pathways in COPD. Eur Respir J 2018;52:OA4922.

99. Graff JW, Powers LS, Dickson AM, et al. Cigarette smoking decreases global microRNA expression in human alveolar macrophages. PLoS One 2012;7:e44066.

100. Savarimuthu Francis SM, Davidson MR, Tan ME, et al. MicroRNA-34c is associated with emphysema severity and modulates SERPINE1 expression. BMC Genomics 2014;15:88.

101.Schembri F, Sridhar S, Perdomo C, et al. MicroRNAs as modulators of smoking-induced gene expression changes in human airway epithelium. Proc Natl Acad Sci U S A 2009;106:2319-24. 